\title{
PERIODIC TRAVELLING WAVES IN A NON-INTEGRABLE ONE-DIMENSIONAL LATTICE***
}

\author{
T. P. VALKERING
}

Dept. of Applied Physics, Twente University of Technology, P.O. Box 217, Enschede, The Netherlands

\begin{abstract}
The existence of a one-parameter family of periodic solutions representing longitudinal travelling waves is established for a one-dimensional lattice of identical particles with nearest-neighbour interaction. The potential is not given in closed form but is specified by only a few global properties. The lattice is either infinite or consists of $N$ particles on a circle with fixed circumference. Waves with low energy are sinusoidal and their properties are studied using bifurcation methods. Waves of high energy, however, are of solitary type, i.e. the excitation is strongly localized.
\end{abstract}

\section{Introduction}

Consider an infinite one-dimensional lattice of identical particles with unit mass that interact through a potential $\tilde{\tilde{V}}=\sum_{n} \tilde{V}\left(r_{n}-r_{n-1}\right)$, where $r_{n}$ is the coordinate of the $n$th particle. This paper is concerned with the existence and with some properties of a particular type of periodic motion

$$
r_{n}(t)=n d_{0}+r(\omega t-n k),
$$

where $d_{0}$ is a constant and $r$ is a function with period $2 \pi$ and zero average. This form represents a travelling wave; $k$ is the wave number, $\omega$ the corresponding angular frequency and $n d_{0}$ is the equilibrium position of the $n$th particle (cf. property (1.2.i) of the potential below). If $k=2 \pi / N, N$ being an integer, the solution (1.1) can be intepreted as a wave in a system consisting of $N$ particles on a circle with fixed circumference $N d_{0}$.

Let $\tilde{V}$ be expressed in terms of a function $V(x), \tilde{V}\left(r_{n}-r_{n-1}\right)=V\left(r_{n}-r_{n-1}-d_{0}\right)$, which has the properties:

(i) $\quad V(x)$ has only one extremum, which is a minimum at $x=0$,

(ii) $\quad V(x)$ is (at least) once continuously differentiable and $V^{\prime}(x)=0$ if and only if $x=0$.

These two properties appear to be sufficient for the existence of a family of solutions of type (1.1), with - loosely speaking - the wave energy as the parameter. In a wellknown case satisfying the above properties, the exponential potential $V(x)=$ $=\beta^{-1} e^{-\beta x}+x($ Toda, 1975$)$, there are exact solutions known of this type. This Toda system, however, is integrable. The aim of the present paper is to tackle the problem for a general, i.e. non-integrable, system.

* Paper presented at the 1981 Oberwolfach Conference on Mathematical Methods in Celestial Mechanics.

** Dedicated to Professor Szebehely, for his stimulating enthusiasm. 


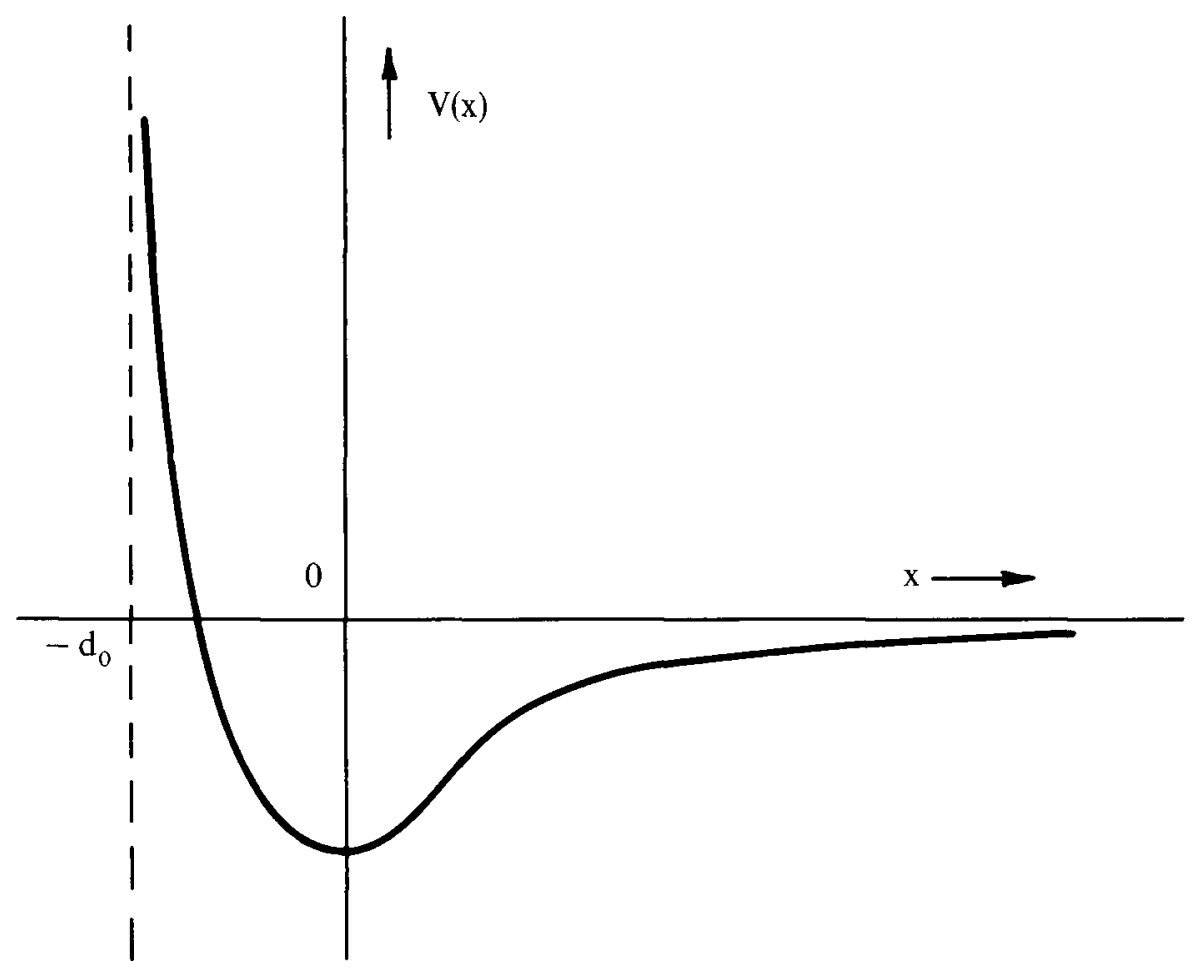

Fig. 1. Typical interaction potential.

The existence question being answered affirmatively, two more assumptions have to be made in order to establish some properties of the solutions.

(i) $\quad V(x)$ is at least three times continuously differentiable,

(ii) $\quad V(x)$ has an asymptote at $x=-d_{0}$ and the singularity is nonintegrable, i.e. the integral $\int_{-d_{0}} V(x) \mathrm{d} x$ does not converge.

A typical example of such a potential (cf. Figure 1) occurs if the force between two particles consists of an attracting and a repelling force, the latter being the stronger for short distances, e.g.

$$
V(x)=\frac{a}{\left(x+d_{0}\right)^{m}}-\frac{b}{\left(x+d_{0}\right)^{n}}+c, \quad a>0, b>0, m>n .
$$

As above, however, the present results depend only on the global properties mentioned above, and not on a particular analytic expression for the potential. The family of solutions of type (1.1) will be shown (for low energy) to bifurcate from the equilibrium state and on the basis of (1.3.i) standard bifurcation methods will be used to establish continuity and differentiability with respect to the parameter. Property (1.3.ii) enables one to formulate some results in the case of high energy. 


\section{Existence}

In this section an outline will be given of the existence proof with emphasis on the most important assumptions. For details we refer to Valkering (1978). The proof is based on critical point theory (cf. Berger, 1977).

Substitution of (1.1) into the Euler-Lagrange equations of the lattice

$$
\ddot{r}_{n}(t)=V^{\prime}\left(r_{n+1}-r_{n}-d_{0}\right)-V^{\prime}\left(r_{n}-r_{n-1}-d_{0}\right)
$$

yields, with $\theta=\omega t-n k$,

$$
\omega^{2} r^{\prime}(\theta)=A V^{\prime}\left(A r^{\prime}(\theta)\right)+C\left(r^{\prime}\right)
$$

where $C\left(r^{\prime}\right)$ is a constant and $A$ denotes the linear transformation defined by

$$
A g(\theta)=-\int_{\theta-k / 2}^{\theta+k / 2} g\left(\theta^{\prime}\right) \mathrm{d} \theta^{\prime}
$$

Differentiation of (2.2) with respect to $\theta$ and substitution of $\theta=\omega t-n k$ leads back to (2.1). Equation (2.2) can be interpreted as an isoperimetric problem in a Hilbert space as will be shown now.

First define a real Hilbert space $H$

$$
H=\left\{s \mid s \in L_{2}[-\pi,+\pi], s(\theta)=s(-\theta), \int_{-\pi}^{+\pi} s(\theta) \mathrm{d} \theta=0\right\}
$$

with inner product

$$
(f, g)=\frac{1}{2 \pi} \int_{-\pi}^{+\pi} f(\theta) g(\theta) \mathrm{d} \theta .
$$

The linear transformation $A$ maps $H$ into itself. Those properties of $A$ that are used in this paper are listed below. The proofs are elementary and not given here (cf. Valkering, 1978).

(i) $A$ is selfadjoint and completely continuous,

(ii) As $(\theta)$ is a continuous function of $\theta$,

(iii) $\sup _{\theta}|A s(\theta)| \leqslant R_{0}^{-1}(k)\|s\|, \quad R_{0}^{-2}(k)=k(2 \pi-k)$,

(iv) the equality in (iii) holds if and only if $s=\alpha s_{0}(\theta), s=\alpha s_{0}(\theta+\pi)$, where $\alpha$ is a constant and $s_{0}(\theta)$ is given by

$$
\begin{aligned}
s_{0}(\theta) & =1 / k, & 0 & \leqslant \theta<k / 2 \\
& =-1 /(2 \pi-k), & k / 2 & <\theta \leqslant \pi .
\end{aligned}
$$




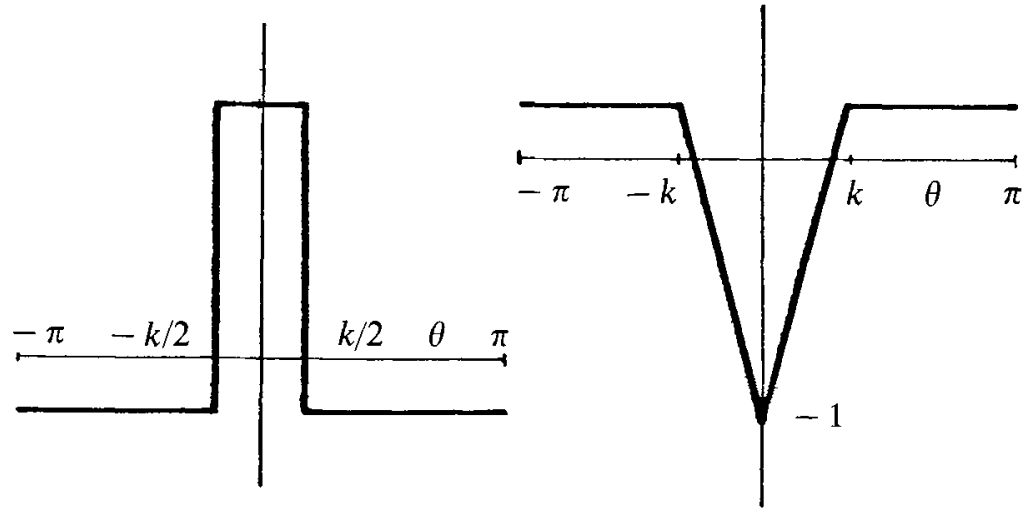

Fig. 2. The functions $s_{0}(\theta)$ and $A s_{0}(\theta)$ for $k=\frac{1}{3} \pi$.

The function $A s$ is then easily evaluated and one finds

$$
\begin{aligned}
A s_{0}(\theta) & =k /(2 \pi-k), & & k \leqslant \theta \leqslant \pi, \\
& =-1+\theta 2 \pi /(k(2 \pi-k)), & & 0 \leqslant \theta \leqslant k .
\end{aligned}
$$

Next define the functional $\mathscr{V}$ on $H$

$$
\mathscr{V}(s)=\frac{1}{2 \pi} \int_{-\pi}^{+\pi} V(A s(\theta)) \mathrm{d} \theta
$$

the domain of definition of which is discussed below. One verifies (cf. Vainberg, 1964; Berger, 1977)

$$
\operatorname{grad} \mathscr{V}(s)=A\left[V^{\prime}(A s)-\frac{1}{2 \pi} \int_{\pi}^{\pi} V(A s(\theta)) \mathrm{d} \theta\right]
$$

With the definition of a second functional

$$
\mathscr{N}=(s, s) / 2, \quad \operatorname{grad} \mathscr{N}=s
$$

it is clear that (2.2) has the form

$$
\omega^{2} \operatorname{grad} \mathscr{N}=\operatorname{grad} \mathscr{V}, \quad s=r^{\prime},
$$

which is an isoperimetric problem.

Before we formulate a theorem concerning the existence of solutions of (2.12), we have to consider the domain of definition of $\mathscr{V}$. One readily sees that the integral in (2.9) exists if

$$
A s(\theta)>-d_{0} .
$$


Assuming $d_{0}$ to be equal to unity it is clear from the assumptions (1.3.ii) for $V(x)$ and and the properties (2.6., ii-iv) of $A$ that $\mathscr{V}(s)$ is defined on a set given by

$$
\begin{aligned}
& \|s\|<R_{0} \quad \text { and } \\
& \|s\|=R_{0} \quad \text { but } \quad s(\theta) \neq s_{0}(\theta), s(\theta) \neq s_{0}(\theta+\pi) .
\end{aligned}
$$

Note that $\left\|s_{0}\right\|=R_{0}$. Obviously $\mathscr{V}$ is defined on all of $H$ if $V(x)$ has no asymptote and is defined for any real value of $x$. The basic result of this section is now formulated in

\section{THEOREM 2.1.}

(i) For any $R, 0<R<R_{0}$, Equation (2.12) has (at least) two solutions, $\left\{\bar{\omega}^{2}(R) ; \bar{s}(R ; \theta)\right\}$ and $\left\{\bar{\omega}^{2}(R) ; \bar{s}(R ; \theta+\pi)\right\}$ with $\|\bar{s}(R ; \theta)\|=\|\bar{s}(R ; \theta+\pi)\|=R$.

(ii) The value of $\mathscr{V}$ at $\bar{s}$ satisfies the inequalities

$$
\begin{array}{lll}
\mathscr{V}(s) \leqslant \mathscr{V}(\bar{s}(R)), & & \|s\|=R, \\
\mathscr{V}(s)<\mathscr{V}(\bar{s}(R)), & & \|s\|<R .
\end{array}
$$

Proof. These results follow from critical point theory (cf. Berger, 1977, in particular Theorem 6.3.7). The conditions to apply this theory are easy consequences of the properties (1.2). A detailed proof is given by Valkering (1978). Further, because $\operatorname{grad} \mathscr{N}$ and $\operatorname{grad} \mathscr{V}$ do not depend explicitly on $\theta$, each solution $\left\{\bar{\omega}^{2} ; \bar{s}(\theta)\right\}$ generates a solution $\left\{\bar{\omega}^{2} ; \bar{s}\left(\theta+\theta_{0}\right)\right\}$. Here we are interested only in the case $\theta_{0}=\pi$, which guarantees that $\bar{s}\left(\theta+\theta_{0}\right) \in H$.

Q.E.D.

Remark 1. If $V(x)$ is defined on the whole real axis, the theorem remains valid but there is no upper bound for $R$.

Remark 2. The above solution is not unique as will be explained in Section 5 . To conclude this section we study the quantity $\mathscr{V}(\bar{s}(R))$. It holds

\section{THEOREM 2.2.}

$\mathscr{V}(\bar{s}(R))$ is an increasing continuous function of $R$ and

$$
\mathscr{V}(\bar{s}(R)) \rightarrow \infty \quad \text { if } R \rightarrow R_{0} .
$$

Proof. The first property is a consequence of (2.15). To prove continuity note that $\mathscr{V}(s)$ is a continuous functional which is a consequence of the continuity of $V(x)$ and of property (2.6. iii) of $A$. Because of (2.15) we have for any $R_{1}$ the inequalities $(\varepsilon>0)$

$$
0 \leqslant \mathscr{V}(\bar{s}(R))-\mathscr{V}(\bar{s}((1-\varepsilon) R)) \leqslant \mathscr{V}(\bar{s}(R))-\mathscr{V}((1-\varepsilon) \bar{s}(R)),
$$

the r.h.s. of which goes to zero if $\varepsilon \rightarrow 0$ because of the continuity of $\mathscr{V}(s)$. Consequently $\mathscr{V}(\bar{s}((1-\varepsilon) R)) \rightarrow \mathscr{V}(\bar{s}(R))$ if $\varepsilon \rightarrow 0$, which has to be proved. Note in this connection 
that neither $\bar{\omega}^{2}(R)$ nor $\bar{s}(R)$ needs to be continuous. Finally we have, again because of $(2.15)$

$$
\mathscr{V}(\bar{s}(R)) \geqslant \mathscr{V}\left(R R_{0}^{-1} s_{0}\right)
$$

which proves (iii) with the non-integrability condition in (1.3.ii).

Because of this theorem $\mathscr{V}(\vec{s}(R))$ is a good parameter to characterize the solution. Moreover, its physical meaning is more clear than that of $R$. The solution of (2.1) corresponding with $\bar{s}$ satisfięs

$$
\bar{r}_{n}(t)-\bar{r}_{n-1}(t)=A \bar{s}\left(\omega t-n k+\frac{1}{2} k\right)+d_{0},
$$

which follows with $s(\theta)=r^{\prime}(\theta)$ and the definition of $A$ (cf. (2.2) and following). Then, however, $\mathscr{V}(\bar{s}(R))$ just equals the quantity

$$
\frac{\omega}{2 \pi} \int_{-\pi / \omega}^{+\pi / \omega} V\left(\bar{r}_{n}(t)-\bar{r}_{n-1}(t)\right) \mathrm{d} t
$$

which is the time average (over one period) of the potential energy in the 'spring' between the $n$th and the $(n-1)$ th particle. Consequently the family of solutions given in Theorem (2.1) covers continuously the complete range (from $V(0)$ to infinity) of potential energies.

\section{The Solution with Low Energy}

In this section standard bifurcation methods are used to establish differentiability and continuity of the solutions $\left\{\bar{\omega}^{2}(R), \bar{s}(R)\right\}$ with respect to $R$ for small values of $R$. The first terms in the Taylor series are given.

With property (2.6.iii) of $A$ and with the Taylor expansion

$$
V(x)=\frac{1}{2} b_{2} x^{2}+O\left(x^{3}\right), \quad b_{2}=V^{\prime \prime}(0),
$$

the analogous relation for $\mathscr{V}$ can be found

$$
\mathscr{V}(s)=\frac{1}{2} b_{2}(A s, A s)+O\left(\|s\|^{3}\right) .
$$

From this expression one may expect that for small $\|s\|$, the solutions of (2.12) are close to those of the analogous problem for the first term in the r.h.s. of (3.2)

$$
\omega^{2} s=\operatorname{grad} \frac{1}{2}\left(b_{2} A s, A s\right)=b_{2} A^{2} s .
$$

The solutions of this linear problem are (verify with (2.3))

$$
\omega^{2}=b_{2} \hat{\omega}_{i}^{2}, s=R \phi_{i}
$$

where

$$
\hat{\omega}_{i}^{2}=\frac{4}{i^{2}} \sin ^{2} i k / 2, \quad \phi_{i}=\sqrt{2} \cos i \theta .
$$


Note that (3.3) is the equation for a lattice with a harmonic interaction potential $V(x)=\frac{1}{2} b_{2} x^{2}$. Writing $\hat{\omega}_{i}^{2}=k^{2} y^{-2} \sin ^{2} y, y=i k / 2$, one verifies easily using the graph of $y^{-2} \sin ^{2} y$ that

$$
\hat{\omega}_{1}^{2}>\hat{\omega}_{i}^{2}, \quad i \geqslant 2 \text {, }
$$

which means in particular that the largest eigenvalue of $A^{2}, \hat{\omega}_{1}^{2}$, is non-degenerate. The solution with $i=1$ realizes the maximum of the functional $b_{2}(A s, A s)$ on the sphere with radius $R$ as is well-known, and so we expect that the solution given in Theorem 2.1 approaches $\left\{\omega^{2}=b_{2} \hat{\omega}_{1}^{2}, R \phi_{1}\right\}$ if $R$ goes to zero. In fact we have

\section{THEOREM 3.1.}

The solutions given in Theorem 2.1 satisfy

$$
\begin{aligned}
& \bar{\omega}^{2}(R)=b_{2} \hat{\omega}_{1}^{2}+O(R), \\
& \bar{s}(R ; \theta)=R \phi_{1}(\theta)+O\left(R^{2}\right) .
\end{aligned}
$$

Proof. With (3.2) one finds

$$
\begin{aligned}
& \mathscr{V}\left(R \phi_{1}\right)=\frac{1}{2} b_{2} \hat{\omega}_{1}^{2} R^{2}+O\left(R^{3}\right), \\
& \mathscr{V}(\bar{s}(R))=\frac{1}{2} b_{2}(A \bar{s}(R), A \bar{s}(R))+O\left(R^{3}\right),
\end{aligned}
$$

and subtracting

$$
\frac{1}{2}\left\{b_{2} \hat{\omega}_{1}^{2} R^{2}-b_{2}(A \bar{s}(R), A \bar{s}(R))\right\}=-\left\{\mathscr{V}(\bar{s}(R))-\mathscr{V}\left(R \phi_{1}\right)\right\}+O\left(R^{3}\right) .
$$

Because of (2.15) we have

$$
\mathscr{V}(\bar{s}(R))-\mathscr{V}\left(R \phi_{1}\right) \geqslant 0
$$

and analogously for the solution $R \phi_{1}$ of (3.3)

$$
b_{2} R^{2} \hat{\omega}_{1}^{2}-b_{2}(A \bar{s}(R), A \bar{s}(R)) \geqslant 0 .
$$

The set $\left\{\phi_{i} \mid i=1,2, \cdots\right\}$ is an orthonormal basis in $H$ and so

$$
\bar{s}(R)=\sum_{i=1}^{\infty} \alpha_{i}(R) \phi_{i}, \quad \sum_{i=1}^{\infty} \alpha_{i}^{2}(R)=R^{2} .
$$

Combination of the results (3.9-11) above shows that there is a positive constant $M$ such that

$$
0 \leqslant b_{2} \hat{\omega}_{1}^{2} R^{2}-b_{2} \sum_{i=1}^{\infty} \alpha_{i}^{2}(R) \hat{\omega}_{i}^{2} \leqslant M R^{3},
$$

which yields after elimination of $\alpha_{1}^{2}$ with (3.12) and with $b_{2}>0$

$$
0 \leqslant \sum_{i=2}^{\infty} \alpha_{i}^{2}\left(\hat{\omega}_{1}^{2}-\hat{\omega}_{i}^{2}\right) \leqslant M R^{3} .
$$


Because $\hat{\omega}_{1}^{2}-\hat{\omega}_{i}^{2}>0$ the final result is

$$
\alpha_{i}^{2}=O\left(R^{3}\right), \quad i=2,3, \ldots, \quad \alpha_{1}^{2}=R^{2}+O\left(R^{3}\right)
$$

from which (3.7b) follows. Substitution of this expression into (2.12) then yields (3.7a), making use of (3.2).

This theorem states that the solution $\left\{\bar{\omega}^{2}(R) ; \bar{s}(R)\right\}$ bifurcates from the trivial solution $\left\{\omega^{2} \in \mathbb{R} ; s=0\right\}$ at $\omega^{2}=\hat{\omega}_{1}^{2} b_{2}$. Consequently bifurcation methods can be used to obtain stronger results for the solutions for small $R$, in particular concerning continuity and differentiability with respect to $R$.

According to general theory, bifurcation of the trivial solution is possible (cf. Temme, 1976, Ch. VII, Th. 1.1) if and only if the linear operator $\omega^{2}-\operatorname{grad}^{\prime} \mathscr{V}(0)$ has no bounded inverse. It holds grad' $\mathscr{V}(0)=b_{2} A^{2}$ and as a result bifurcation may occur only if $\omega^{2}$ equals one of the eigenvalues of $b_{2} A^{2}, b_{2} \hat{\omega}_{i}^{2}$. Because of Theorem 3.1 we are interested in bifurcation at $\omega^{2}=b_{2} \hat{\omega}_{1}^{2}$. The multiplicity of this eigenvalue being unity (cf. (3.6)), the analysis of the bifurcation is rather simple and the following result holds.

\section{THEOREM 3.2 .}

(i) There is an $\varepsilon_{0}>0$ and a unique solution $\left\{\omega^{2}(\varepsilon) ; s(\varepsilon)\right\}, 0 \leqslant \varepsilon \leqslant \varepsilon_{0}$, that is continuous with respect to $\varepsilon$ and that satisfies

$$
\begin{aligned}
& s(\varepsilon)=\varepsilon \phi_{1}+v(\varepsilon), \quad v \perp \phi_{1} \text { and }\|v\|=O\left(\varepsilon^{2}\right), \\
& \omega^{2}(\varepsilon)=b_{2} \hat{\omega}_{1}^{2}+O(\varepsilon) .
\end{aligned}
$$

(ii) If $V(x)$ is $(k+1)$ times continuously differentiable, $k \geqslant 2$, then $\omega^{2}(\varepsilon)$ and $s(\varepsilon)$ are $k$ times continuously differentiable with respect to $\varepsilon$.

(iii) The solutions satisfy

$$
\omega^{2}(\varepsilon)=\omega^{2}(-\varepsilon), \quad s(\varepsilon ; \theta)=s(-\varepsilon ; \theta+\pi) .
$$

Proof. With property (1.3.i) of $V(x)$, i.e. $V(x)$ is three times continuously differentiable, one derives (see also (2.6)), that grad $\mathscr{V}$ is twice continuously differentiable and (i) follows with Berger (1977, Section 4.1C). The order relations (3.16) follow with Berger (1977, Section 4.1 D), after showing that there exists a positive constant $M$ such that

$$
\begin{aligned}
\|\{\operatorname{grad} \mathscr{V}(s) & -\operatorname{grad} \mathscr{V}(0) s\}-\left\{\operatorname{grad} \mathscr{V}\left(s^{\prime}\right)-\operatorname{grad} \mathscr{V}(0) s^{\prime}\right\} \| \leqslant M\{\|s\|+ \\
+ & \left.\left\|s^{\prime}\right\|\right\}\left\|s-s^{\prime}\right\|,
\end{aligned}
$$

which can be proved with the aid of Taylor's theorem. In order to prove part (ii) note that $\operatorname{grad} \mathscr{V}$ is $k$ times continuously differentiable if $V(x)$ is $k+1$ times continuously differentiable. The result (ii) is then a consequence of the existence proof 
in (i) that makes use of the implicit function theorem. A detailed proof is given by Temme (1976, Ch. VII, Section 3) where also proofs for (i) are given. Part (iii) is a consequence of the uniqueness mentioned in (i). If $\left\{\omega^{2}(\varepsilon) ; s(\varepsilon ; \theta)\right\}$ is a solution then obviously $\left\{\hat{\omega}^{2}=\omega^{2} ; \hat{s}(\theta)=s(\theta+\pi)\right\}$ is also a solution. Writing this solution as a function of $\hat{\varepsilon}=\left(\hat{s}, \phi_{1}\right)$, one finds with $\hat{\varepsilon}=\left(s(\theta+\pi), \phi_{1}\right)=-\varepsilon$

$$
\hat{\omega}^{2}(\hat{\varepsilon})=\omega^{2}(-\hat{\varepsilon}), \quad \hat{s}(\hat{\varepsilon} ; \theta)=s(-\hat{\varepsilon} ; \theta+\pi) .
$$

Equation (3.17) then follows from the uniqueness of the solution $\left\{\omega^{2}(\varepsilon), s(\varepsilon)\right\}$.

Q.E.D.

The solutions $\left\{\bar{\omega}^{2}(R) ; \bar{s}(R)\right\}$ and $\left\{\omega^{2}(\varepsilon) ; s(\varepsilon)\right\}$ both bifurcate from the trivial solution $s=0$ at $\omega^{2}=b_{2} \hat{\omega}_{1}^{2}$. The uniqueness result of Theorem 3.2.(i) then implies that one must be able to identify them.

THEOREM 3.3. There exists a $k$ times continuously differentiable function $\tilde{\varepsilon}(R)$ with the properties

$$
\tilde{\varepsilon}(R)=-\tilde{\varepsilon}(-R), \quad \tilde{\varepsilon}(R)=R+O\left(R^{3}\right)
$$

that relates the solutions of Theorem 2.1 with those of Theorem 3.2 as follows

$$
\begin{aligned}
& \bar{\omega}^{2}(R)=\omega^{2}(\tilde{\varepsilon}(R)), \\
& \bar{s}(R, \theta)=s(\tilde{\varepsilon}(R) ; \theta), \quad \bar{s}(R ; \theta+\pi)=s(-\tilde{\varepsilon}(R) ; \theta) .
\end{aligned}
$$

Proof. Define the function

$$
\widetilde{R}(\varepsilon)=\varepsilon \sqrt{1+\varepsilon^{-2}\|v(\varepsilon)\|^{2}},
$$

$v(\varepsilon)$ being given in (3.16). As a consequence of Theorem (3.2), $\tilde{R}(\varepsilon)$ is $k$ times continuously differentiable with respect to $\varepsilon$ and $\tilde{R}(\varepsilon)=-\tilde{R}(-\varepsilon)$. One verifies that $\mathrm{d} /(\mathrm{d} \varepsilon) \tilde{R}(\varepsilon)_{\varepsilon=0}$ equals unity and the implicit function theorem (cf. Dieudonné, 1969, Ch. X.2) guarantees the existence of a $k$ times continuously differentiable inverse function $\tilde{\varepsilon}(R)$, defined in some neighbourhood of $R=0$. The properties (3.20) then follow trivially. With (3.16) and (3.22) one finds $\|s(\tilde{\varepsilon}(R) ; \theta)\|=\|s(\tilde{\varepsilon}(R) ; \theta+\pi)\|=R$ and because of the uniqueness (3.21) follows.

This theorem justifies the calculation of the Taylor expansions around $R=0$ of $\bar{\omega}^{2}(R)$ and $\bar{s}(R ; \theta)$, which are of the form

$$
\begin{aligned}
\bar{\omega}^{2}(R) & =b_{2} \hat{\omega}_{1}^{2}\left\{1+\gamma R^{2}+\cdots,\right. \\
\bar{s}(R) & =R \phi_{1}+R^{2} \sigma_{2}+R^{3} \sigma_{3}+\cdots .
\end{aligned}
$$

To find the quantities $\gamma, \sigma_{2}, \sigma_{3}, \cdots$ these expressions are substituted into (2.12). After lengthy but straight forward calculations, one finds, comparing coefficients 
of equal order

$$
\begin{aligned}
\gamma & =\frac{1}{4} \hat{\omega}_{1}^{2}\left[\left(\frac{b_{3}}{b_{2}}\right)^{2} \frac{\hat{\omega}_{2}^{2}}{\hat{\omega}_{1}^{2}-\hat{\omega}_{2}^{2}}+\frac{b_{4}}{b_{2}}\right], \quad b_{i}=V^{(i)}(0), \\
\sigma_{2} & =\beta_{22} \phi_{2}, \quad \beta_{22}=-\frac{1}{4} \sqrt{2} \frac{b_{3}}{b_{2}} \frac{\hat{\omega}_{1}^{2} \hat{\omega}_{2}}{\hat{\omega}_{1}^{2}-\hat{\omega}_{2}^{2}}, \\
\sigma_{3} & =\beta_{31} \phi_{1}+\beta_{33} \phi_{3}, \quad \beta_{31}=-\frac{1}{2} \beta_{22}^{2}, \\
\beta_{33} & =\frac{1}{12} \frac{\hat{\omega}_{1}^{3} \hat{\omega}_{3}}{\hat{\omega}_{1}^{2}-\hat{\omega}_{3}^{2}}\left[3\left(\frac{b_{3}}{b_{2}}\right)^{2} \frac{\hat{\omega}_{2}^{2}}{\hat{\omega}_{1}^{2}-\hat{\omega}_{2}^{2}}+\frac{b_{4}}{b_{2}}\right] .
\end{aligned}
$$

The calculations are somewhat simplified if one makes use of $(\bar{s}(R), \bar{s}(R))=R^{2}$ and $\bar{s}(R ; \theta)=\bar{s}(-R ; \theta+\pi)$ which imply respectively

$$
\begin{aligned}
\left(\phi_{1}, \sigma_{2}\right) & =0, \quad 2\left(\phi_{1}, \sigma_{3}\right)+\left(\sigma_{2}, \sigma_{2}\right)=0, \\
\left(\sigma_{2}, \phi_{2 i-1}\right) & =\left(\sigma_{3}, \phi_{2 i}\right)=0, \quad i=1,2, \cdots .
\end{aligned}
$$

\section{The Solution with High Energy}

According to Theorem 2.1 the solution of Equation (2.12) represents a maximum of $\mathscr{V}$ on a surface of constant $\mathcal{N}$, i.e. on a sphere $S_{R}$ with radius $R$ around the origin. Now note that on the sphere with radius $R_{0} \mathscr{V}$ is bounded everywhere, apart from the points $s_{0}(\theta)$ and $s_{0}(\theta+\pi)$ where $\mathscr{V}$ is singular (cf. (2.14)), in particular $\mathscr{V}(s) \rightarrow \infty$ if $s \rightarrow s_{0}$ along the trajectory $s=\alpha s_{0}, \alpha \rightarrow 1$. Then one might expect that if $R$ is close to $R_{0} \mathscr{V}$ attains its maximum on $S_{R}$ at a point close to $s_{0}$ (cf. Figure 3). More precisely it holds

THEOREM 4.1. For any $\varepsilon>0$ there is $a \delta>0$ such that

$$
\left\|\bar{s}(R)-s_{0}\right\|<\varepsilon R_{0}, \sup _{\theta}\left|A \bar{s}-A s_{0}\right|<\varepsilon, \quad \text { if } \quad R_{0}-R<\delta R_{0} .
$$

Furthermore there is an $M>0$ independent of $\varepsilon$ such that

$$
\bar{\omega}^{-2}(R) \leqslant M / V(-1+\varepsilon) \text {, if } R_{0}-R<\delta R_{0} .
$$

Proof. Cf. Valkering (1978, Section 6). Note that $d_{0}$ equals unity.

If we define $\bar{s}\left(R_{0}\right)=s_{0}$ and $\bar{\omega}^{-2}\left(R_{0}\right)=0$ then this theorem states that at $R_{0} \bar{s}(R)$ is continuous in $L_{2}$-norm, that $A \bar{s}(R)$ is continuous in supremum norm and that $\bar{\omega}^{-2}$ is continuous. Because of the fact however that $\mathscr{V}(s)$ is singular at $s_{0}$ it is difficult to extend the above results to continuity in a neighbourhood of $R_{0}$, analogous to the results of Section 3 for a neighbourhood of $R=0$.

In order to interprete this high energy limit note that the velocity of the $n$th particle 


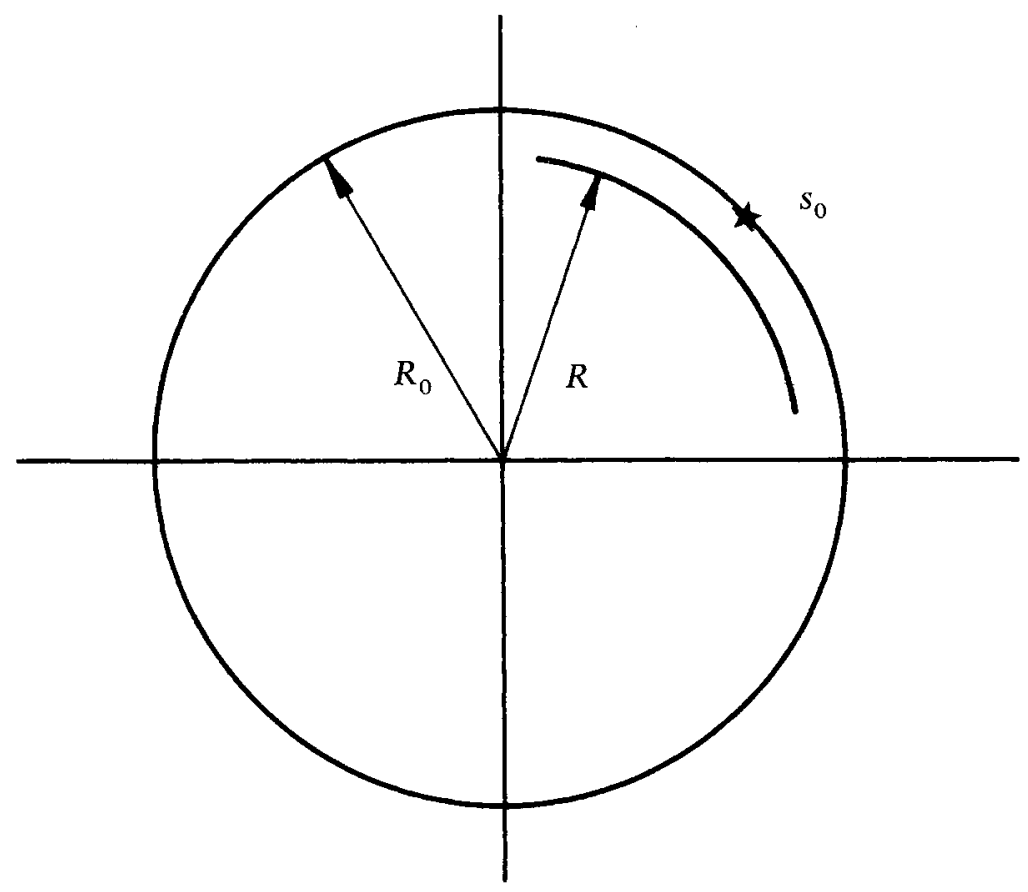

Fig. 3. Two-dimensional representation of the Hilbert space $H$.
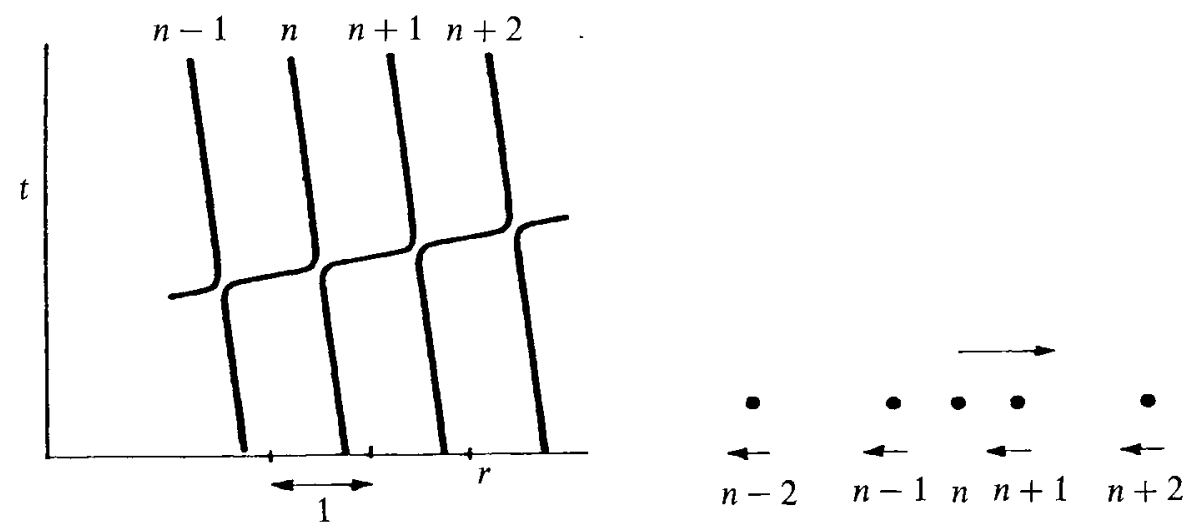

Fig. 4. Coordinate-time diagram of a wave in a hard sphere chain with velocities $\dot{r}_{n}(t)$ proportional to $s_{0}(\omega t-n k)$.
Fig. 5. Schematic representation of a wave in a hard sphere chain. The arrows denote the velocities of the corresponding particles. 
is given by

$$
\dot{r}_{n}(t)=\omega s(\omega t-n k) \text {. }
$$

Replacing $s$ here and in (2.19) by $s_{0}$ one readily sees that this limiting case represents a wave in a hard sphere lattice as is illustrated in Figures 4 and 5. Note in this connection that we are dealing with a periodic wave in an infinite lattice with wavenumber $k$ and wavelength $\lambda=2 \pi / k$. If $k=2 \pi / N, N$ being a positive integer, the quantity $r(\omega t-n k)$ in (1.1) is periodic in $n$ with period $N$ and consequently it is also a solution for a closed circular chain with $N$ particles. If $\left(R_{0}-R\right) / R_{0}$ is sufficiently small $\left(R \neq R_{0}\right)$ the solution represents either an infinite set of equidistant localized compressions that run through the infinite lattice with high velocity or one local compression going around the $N$-particle chain.

\section{Concluding Remarks}

We have seen that for low values of $R$ the wave is sinusoidal. Note that its phase velocity, being given by $k^{-1} \bar{\omega}(R)$, may increase or decrease as $R$ increases according to the sign of $\gamma$ (cf. (3.23)). In the other limiting case, $R \rightarrow R_{0}$ we have a strongly localized compression wave. It is an open question, however, what the wave looks like for intermediate values of $R$. It is not even known if $\bar{\omega}^{2}(R)$ and $\bar{s}(R)$ are continuous as a function of $R$, let alone in which way the sinusoidal wave transforms into the peaked form if $R$ increases. One may hope that some results may be obtained for the types of potential functions $V(x)$ we are dealing with, possibly with some additional restrictions.

Finally we consider the uniqueness of the solution. $\mathscr{V}$ having a global maximum on the sphere $S_{R}$ at $\bar{s}(R)$ (cf. (2.15a)), one expects the existence of other stationary points of $\mathscr{V}$, not representing global maxima. Indeed Ljusternik-Schnirelman critical point theory can be applied to guarantee the existence of an infinite set of. solutions (cf. Berger, Theorem 6.6.11). Such a set can be constructed on the basis of Theorem 2.1 as will be explained now. Consider some basic wave number $k_{0}$ and solve (2.12) for each wave number $k=i k_{0}, i=1,2, \cdots$, according to Theorem 2.1. As a result one finds a set of travelling waves in the lattice with wave numbers $i k_{0}$. Such a wave, however, can be written in the form (1.1) with $k=k_{0}$, but $r$ having a smaller period $2 \pi / i$. Consequently it must be possible to construct an infinite set of solutions of (2.12) with $k=k_{0}$ on the basis of the Theorem 2.1 solutions of (2.12) with $k=i k_{0}$. This appears to be possible, and some calculations show that if $\bar{\omega}^{2}(R ; k)$ and $\vec{s}(R, k ; \theta)$ denote the solution of (2.12) for general values of $k$, an infinite set of solution of (2.12) for $k=k_{0}$ is given by

$$
\begin{aligned}
& \bar{\omega}_{i}^{2}\left(R ; k_{0}\right)=i^{-2} \bar{\omega}^{2}\left(i^{-1} R ; i k_{0}\right), \\
& \bar{s}_{i}\left(R, k_{0} ; \theta\right)=i \bar{s}\left(i^{-1} R, i k_{0} ; i \theta\right), \quad i=1,2, \ldots
\end{aligned}
$$


This result is verified easily if the interaction force is linear, i.e. if $V(x)=\frac{1}{2} b_{2} x^{2}$. In that case the above set describes all solutions of (2.12) with $k=k_{0}$. It remains an open question, however, if this holds true in the case of the present non-linear potential.

\section{References}

Berger, M. S. : 1977, Nonlinearity and Functional Analysis, Academic Press, New York.

Dieudonné, J. : 1969, Foundations of Modern Analysis, Academic Press, New York.

Temme, N. M. (ed.): 1976, Nonlinear Analysis, MC Syllabus 28, Mathematisch Centrum, Amsterdam. Toda, M. : 1975, 'Studies of a Non-linear Lattice', Phys. Rep. 18, 1-124.

Vainberg, M. M. : 1964, Variational Methods for the Study of Nonlinear Operators, Holden Day, San Francisco.

Valkering, T. P. : 1978, 'Periodic Permanent Waves in an Anharmonic Chain with Nearest-neighbour Interaction, $J$. Phys. A 11, 1885-1897. 